\title{
Human Action Adverb Recognition: ADHA Dataset and A Three-Stream Hybrid Model
}

\author{
Bo Pang, Kaiwen Zha, Cewu Lu * \\ Shanghai Jiao Tong University, China \\ pangbolsjtu.edu.cn, Kevin_zhalsjtu.edu.cn, lucewulcs.sjtu.edu.cn
}

\begin{abstract}
We introduce the first benchmark for a new problem recognizing human action adverbs (HAA): "Adverbs Describing Human Actions" (ADHA). We demonstrate some key features of ADHA: a semantically complete set of adverbs describing human actions, a set of common, describable human actions, and an exhaustive labeling of simultaneously emerging actions in each video. We commit an in-depth analysis on the implementation of current effective models in action recognition and image captioning on adverb recognition, and the results show that such methods are unsatisfactory. Moreover, we propose a novel three-stream hybrid model to deal the HAA problem, which achieves a better result.
\end{abstract}

\section{Introduction}

Computer vision aims to recognize semantic labels in visual data (e.g., images, video). We find these semantic labels are inside our language system. For example, object detection/recognition [27, 26] can be considered as exploring "noun" in visual data. To understand "verb", action recognition [5, 36, 30, 39, 14, 13, 14] has been extensively studied. Moreover, the "Adjective" labels (e.g., cool, dark, beautiful) are explored by attribute learning [25]. Until now, most of computer vision researchers have ignored an important kind of words - "Adverb", which can express the attitude and mood of the subject, and the attributes of the action as well. From the viewpoint of language research [35], these concepts convey more sensitive semantics compared with actions and nouns.

If we can teach an AI to understand adverbs of an action, it implies that the AI can understand the attitude and mood of the action player, which is necessary for interactive robots. We also believe this is the preliminary work to make AI understand the purpose and the intent behind actions.

We are the first one to explore the topic of human action adverbs (HAAs) recognition. Unlike action recogni- tion, HAAs describe the conceptions with very sensitive visual patterns that are difficult to recognize. For example, is the drinking people happy or sad; Is the hand shaking an expression of excitement or politeness? Extensive experiments show that understanding adverb is very challenging to current state-of-the-art deep learning architecture. Note that in the topic of image captioning [38], adverb may be included in the language material. However, on one hand, they don't take adverb as a target. On the other hand, we believe adverb recognition will be one of the most important tools to further advance the topic of image captioning.

By now, we have made tremendous progress in action recognition. Many large-scale datasets like UCF101 [29], YouTube-8M [1] are built and some excellent models like two-stream network [28] et al. have been proposed. Despite significant advances in action recognition, HAAs, as an important attribute is a missing study. There is no appropriate dataset for HAA recognition.

Similar to other beginnings of new topics, we built a video dataset with adverbs labeled for actions. There are 12000 videos in total covering 350 action-adverb pairs (e.g., "smoking sadly") over 32 actions described by 51 adverbs. There are three kinds of HAAs that can respectively describe the subject's mood, attitude and the attributes of the actions. We commit a social experiment to make sure that the HAAs space can describe almost all the meanings a human want to express after seeing a short action video.

We highlight four features of the dataset. First, an average of 11 distinct HAAs per action (no dull action which can only be labeled by few adverbs). Second, our adverb categories are based on semantics rather than words. It means that we do not take "smoke sadly" and "smoke sorrowfully" as different categories. Third, the dataset is multi-labeled. An action can be labeled with multiple HAAs describing mood, attitude and action attributes simultaneously. Fourth, each video is labeled by three annotators, with different backgrounds to reduce bias. Accordingly, a novel evaluation metric is designed. Fig. 1 shows example video frames and annotations in ADHA.

We prove that this dataset is able to act as a benchmark 


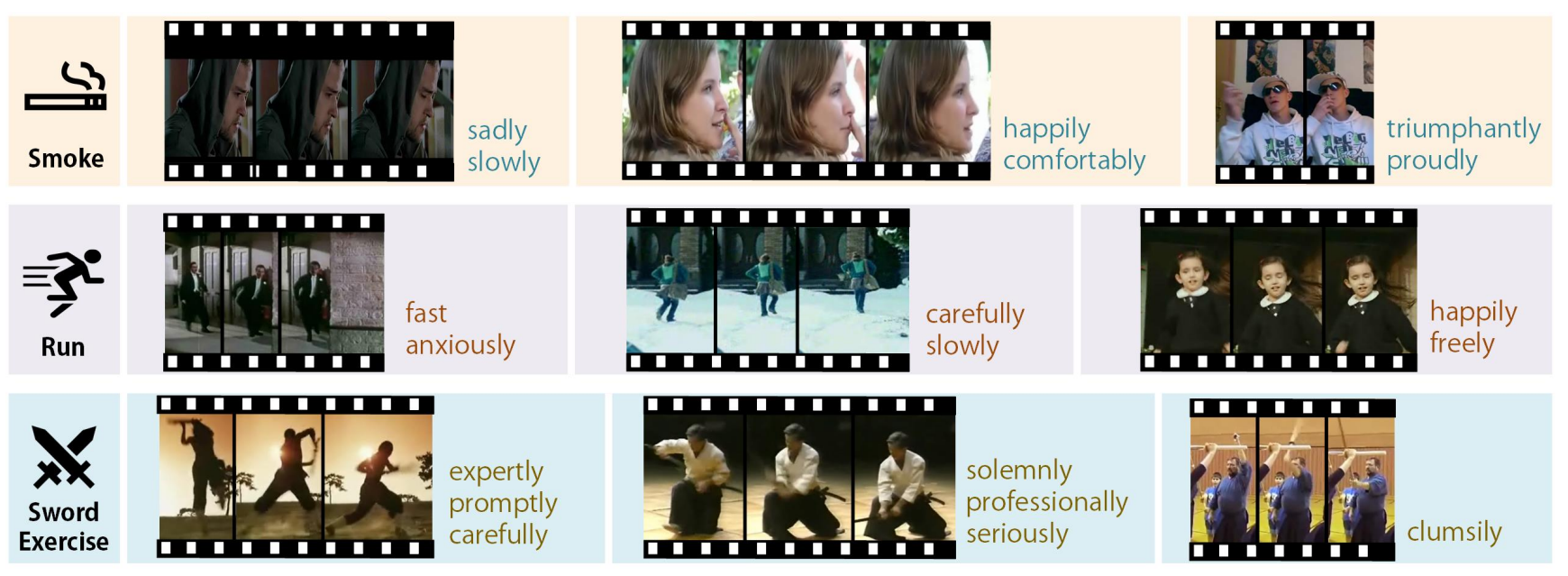

Figure 1. Example frames and annotations in ADHA.

of the HAA recognition problem. We commit experiments to answer the following questions: First, how well can the current action recognition and image captioning approaches deal with the HAA recognition problem? Second, can pose help to understand HAA? Third, can we use expression knowledge as extra information to solve the HAA recognition problem?

The contributions of this work are that: 1) we build a large-scale video dataset labeled with actions, HAAs, and human instance boundingboxes. 2) We benchmark several current action recognition, pose estimation, and image captioning models on ADHA. 3) We propose a hybrid model using pose, expression, optical flow and RGB information and achieve a relative better performance.

\subsection{Related Dataset}

To our knowledge, we are the first to study HAA recognition. Building high-quality benchmark dataset is the first step to explore this topic. Therefore, in this section, we focus on investigating some related datasets.

Action Dataset Action recognition in video has made great progress due to many excellent datasets, from small simple datasets like KTH [18] to large-scale, real-world datasets such as Youtube-8M [1], UCF-101 [29], and Sport$1 \mathrm{M}$ [14]. However, action is not only contained in video. In other words, people as well as AI can tell the action by only one image. Sometimes, recognition of an action in videos stems from the recognition of a related object in the scenario. For example, a model might recognize the action of swimming by recognizing the swimming pool. Actually, the model does not understand, or rather, pay attention to the action itself. Instead, it solves an object detection problem. However, if a model can recognize adverbs very well, we can tell that the model has the ability to understand the action, actions' attributes and the player's attitude. This is why HAA recognition is important and difficult.

Video Captioning Dataset Video captioning is a hot issue and there are many datasets designed for it, for example MSR-VTT [37] which is built from the queries on a commercial video search engine and covers $10 \mathrm{k}$ clips, YouTube2Text [11] with 2,000 video snippets and $120 \mathrm{~K}$ sentences, ActivityNet Captions [15], a large-scale benchmark for dense-captioning events which contains $20 \mathrm{k}$ videos amounting to 849 video hours with $100 \mathrm{k}$ total descriptions. Although video captioning needs adverbs, it needs to concern many other things like fixed phrases and idioms, which is biased against our goal. And in our HAA set, we have removed the synonyms, because we do not want to discriminate them. While in video captioning, they need to be taken into account. Therefore, although there are many datasets built for video captioning, they are not suitable for HAA recognition.

Human Expression Dataset Face expressions can convey the mood and attitude of human like HAAs. And many datasets are built for it like Emotiw2016 [4] which contains an audio-video based emotion and a group-based emotion recognition sub-challenges, MMI [32] — a resource for building and evaluating facial expression recognition algorithms, HUMAINE [6] which provides naturalistic clips recording pervasive emotion and suitable labeling techniques. But there are also some shortcomings. The expression recognition mainly deals with faces in images or videos, but HAA recognition needs to recognize subject's mood and attitude from his action not merely face expression. Moreover, adverb recognition can tell the attributes of 
the action like how fast, heavily, which is beyond the expression recognition. Therefore, we need to build a HAA recognition dataset to solve this much more difficult and comprehensive problem.

\section{Constructing ADHA}

Our dataset is constructed for recognizing adverbs describing human actions (HAA). First, we introduce how to make the action and adverb list. Then, videos are collected based on the given lists. The pipeline of the annotation is shown in Fig. 2. Finally, we present the annotation details on the collected videos.

\subsection{Selecting Action and Adverb Categories}

Adverbs are used to describe the actions, so we fix the action categories list first. Then make the adverb list according to the given selected action categories.

Action Collection Previous action dataset [1, 18, 29, 14] constructors seek to build sets that cover most of action categories, those frequently happen in daily life. So, in this paper, we adopt the union set of action categories from these datasets as our action candidates shortlist. As a research dataset, we expect the selected actions to require adverb descriptions. For example, in common cases some sport actions such as fencing, gymnastics, swimming are without attitude and mood. So those adverb-needless actions are not our target. To further refine the shortlist, we turn to language prior knowledge. We extract about 0.2 million video descriptions as our language materials. Then we rank action categories by the percentage of being described by at least one adverb. According to this score, we finally choose top 32 actions in the shortlist: $\{$ brush hair, chew, clap, climb stairs, dive, draw sword, drink, eat, fall floor, hit, hug, kick, kiss, pick, pour, pullup, punch, push, run, shake hands, shoot bow, shoot gun, sit, smoke, stand, swing baseball, sword, sword exercise, talk, throw, walk, wave .

Adverb Collection Given action categories, we build the list of the adverbs. We consult the word frequency from the Corpus of Contemporary American English (COCA) which is an authoritative corpus of American English [2]. From thousands of adverbs, we choose 113 adverbs which are able to describe actions and possess the highest word frequencies. After removing the synonyms, there are 51 adverbs left. In order to make sure that these 51 adverbs cover all the meanings a person wants to express after seeing a short action video, we conduct a user study. We invite 50 students with different majors from the college and give each of them 50 videos and the adverb set. They need to watch each video first and then check whether the adverb set can cover what they want to express about the video.



Figure 2. Pipeline for collecting and annotating the videos. We take "kiss" action as an example.

From the result, we find that for male, the adverb set can cover $98.8 \%$ requirements and for female it is $97.4 \%$.

Adverb-action Pair Collection With 32 actions and 51 adverbs, we then group adverb-action pairs. In this process we take a $\mathrm{N}$-gram data 1 as the reference. This $\mathrm{N}$-gram data is better than Google N-Gram for us, since it has a large corpus (COCA) and takes the low frequency (even only appear 1 or 3 times) n-grams into account. This n-gram data provides us the frequencies of action-adverb pairs. Then we obtain the candidate action-adverb pairs set on the basis of the frequencies. For every action there are around 11 appropriate adverbs. In total we have 350 adverb-action pairs. We will list the adverb and adverb-action pairs in supplementary file.

\subsection{Video Collection and Annotation}

Video Collection We collect video clips from both YouTube and existing action datasets. First, videos from several existing datasets HMDB51 [17], HOHA [19], UCF101 [29] are used. Then, we add videos from YouTube in order to deal with the long tail problem on adverbs. The details of video collection will be illustrated in section 2.3 . Each human instance in the video preforms one action only.

Human Instance Annotation Our annotation is in human instance level, because we should know who performs the action. What's more, some actions like "kiss" and "hug" have more than one player, so that we need to annotate them respectively. We propose a semi-automatic annotation framework to effectively localize human instance. We label the human boundingbox at the first frame and use

\footnotetext{
${ }^{1}$ https://www.ngrams.info/
} 


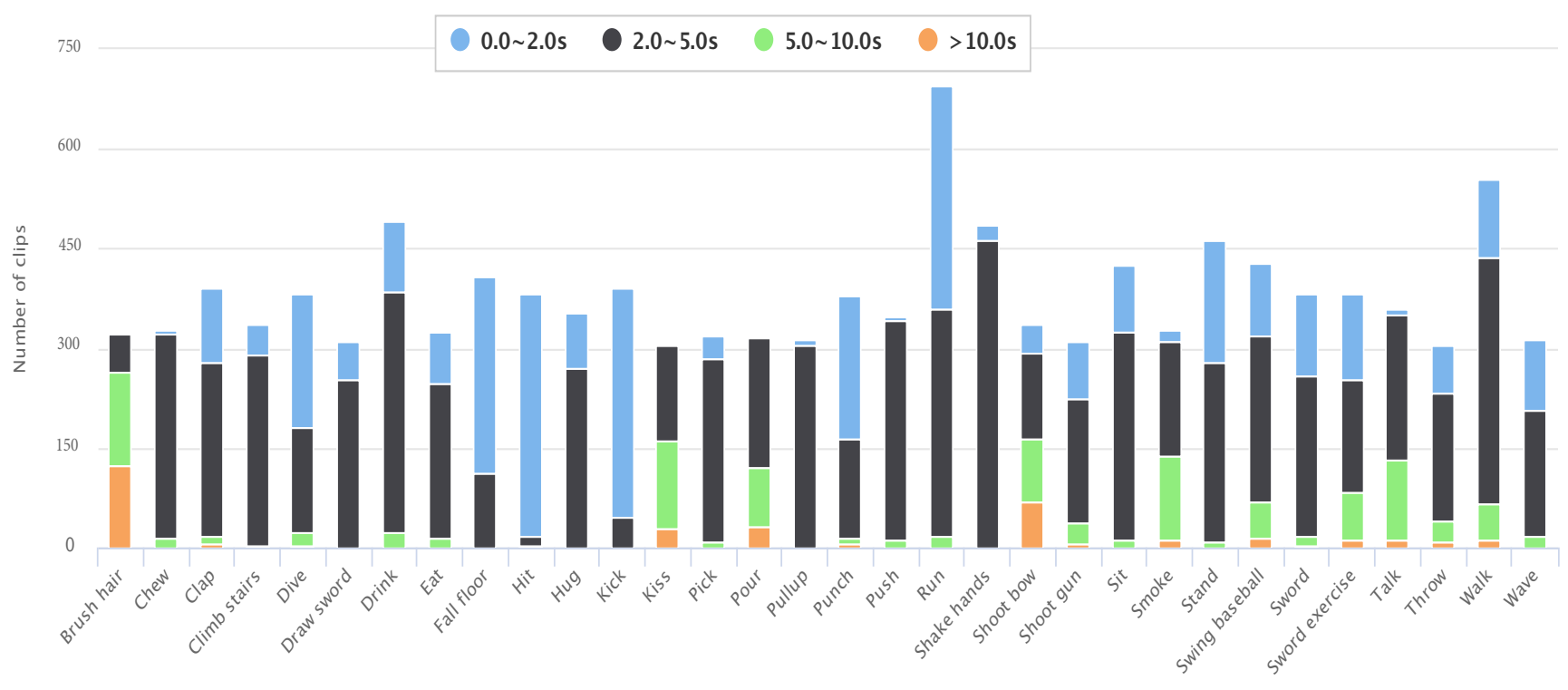

Figure 3. Number of videos per action. The distribution of video durations is illustrated by the colors.

object tracking model MDNet [21] which is the winner of the VOT-2015 challenge [16] to search corresponding human instance in the following frames. To improve the robustness, we implement human detection (using FasterRCNN [10]) to revise tracking bounding box. In detail, we pick up a human detection box that is the closest to the tracking result box based on IOU overlap criterion, then average them as revised result. Annotators observe the automatic video annotation on-line. If the automatic annotation is inaccurate, the annotator need to stop the video and manually correct the bounding boxes. In this way, we only need to annotate some key frames, instead of all frames.

Annotator We invite 100 annotators with different ages, genders and nationalities. Since our adverbs are presented in English, all the annotators are either native English speakers or excellent English speakers.

Annotation Interface Our interface is friendly. We play video with labeled human instance boxes. Then, the system gives out an adverb list to choose from. Annotators can select one or multiple adverbs to describe the observed action video, and they can replay it many times until they are confident enough with their choices. We don't set any time limitation for them.

Work Assign Adverbs are used to describe mood, attitude like concepts which are more subjective than object and action category labeling. Annotating by only one person may not cover all the feelings of people. Therefore, each video is assigned to three different annotators. We make sure those three annotators should have diverse backgrounds (e.g., nationality, age, gender, education background). We find three annotations for each action instance is enough to cover most of feelings. A study experiment is conducted: After annotation is done, we randomly sample 1000 action instances and re-label them by 30 new annotators outside those 100 annotators. We find that $96.3 \%$ cases have at least one original annotation which is exactly the same with the new one.

\subsection{Dataset Statistics and Discussion}

In total, there are 32 actions, 51 adverbs, 350 actionadverb pairs, and 12000 videos of ".avi" in ADHA. A video may be annotated with more than one adverb and the average number of adverbs per video is 1.81 . Some statistics about the videos are shown in Tab. 1. We can tell that the dataset contains only short videos, which reduces the difficulty. For every action and adverb, we count the amount of the videos about them and show the distributions in Fig. 3 and Fig. 4. To deal with the long tail problem, we recollect many videos. For example, before recollection, "hug sadly" only has 9 samples in 483 videos. While after recollection, it has 30 samples in 510 videos. To get the diversity among the 3 annotations, we use a 51-dimension vector to represent the annotation, then calculate the average of the Manhattan distances between every two of the 3 annotators. Using $a_{v, i}$ to represent the $i$-th annotation of the video $v$, then the diversity $d$ can be written as:

$$
\left.d=\operatorname{avg}_{v}\left(\sum_{i<j}\left|a_{v, i}-a_{v, j}\right|\right]\right) .
$$

The diversity of ADHA annotation is 1.376 . 


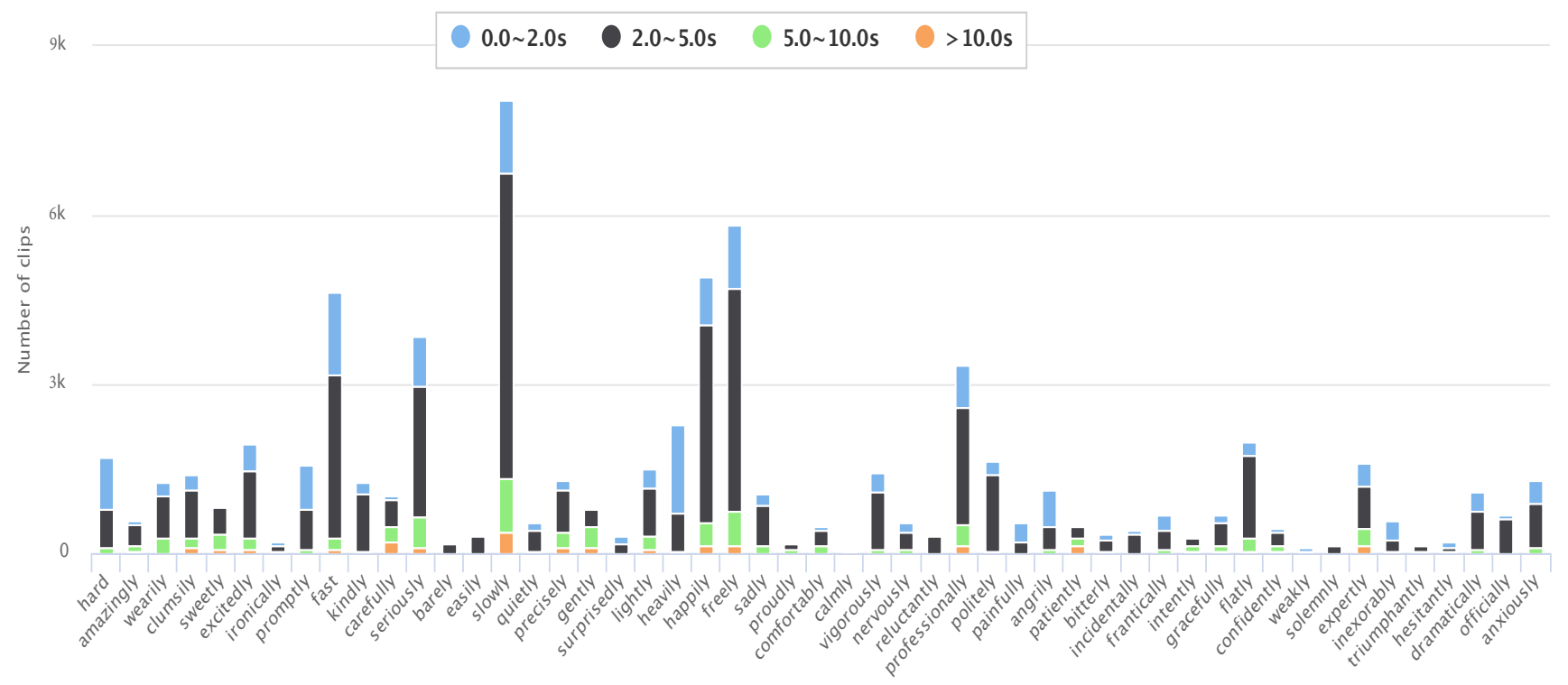

Figure 4. Number of videos per adverb. The distribution of video durations is illustrated by the colors.

\begin{tabular}{|c|c|}
\hline \#Actions & 32 \\
\#Adverbs & 51 \\
\#Clips & 12000 \\
Mean Clip Length & $3.25 \mathrm{~s}$ \\
Total Duration & $43071 \mathrm{~s}$ \\
\hline
\end{tabular}

Table 1. Statistics about the videos

\section{Benchmark System}

In this section, we evaluate several representative approaches designed for action recognition and image captioning using our ADHA dataset and analyze the influence of the expression knowledge on HAA recognition. First, we will briefly introduce the prior approaches.

\subsection{Related work}

Action Recognition: We have made great progress in action recognition and many excellent models have been proposed. Prior works have explored various strategies. Some works extract features of video frames and then fuse them. RNN is widely used to fuse [5, 36, 30] and many pooling methods have been developed [39, 14]. CNN gains great success in image processing so that many CNN models in video field appear like 3D-CNN [13] and time dimension convolution [14]. Whereas other jobs take other methods to deal with the temporal information like optical flow [28], trajectories [33], and human pose estimation [20]. All of these methods do good job in action recognition on video. Can they deal with the HAA recognition problem as well?

Face Expression: Adverbs convey mood and attitude, which can also be inferred by face expression. Nowadays there are many outstanding expression recognition models like [7] which uses a C3D, CNN, RNN hybrid networks, and [23] which uses transfer learning to deal with small datasets.

Pose estimation: For pose estimation, we usually output the heat map of the key points or the skeleton. In [22] a stacked hourglass network is used. And AlphaPose [8] consists of three components: Symmetric Spatial Transformer Network (SSTN), Parametric Pose NonMaximumSuppression (NMS), and Pose-Guided Proposals Generator (PGPG).

Image Captioning: Image captioning also needs adverbs. In [24] a graph based method is used. In [38] the authors add visual attention to the model.

\subsection{Experiment Setup}

Metric We choose mean average precision (mAP) and Hit@k as the evaluation metrics because a video can be labeled with more than one adverb and the adverbs are not exclusive between each other. On the other hand, mAP and Hit@k are widely used metrics for action recognition and have been used in many benchmarks [29, 1, 14], which will give people a better intuitive feeling on the metric values.

mAP: Given a video, a model will give a classification score for every adverb. Then we compute average precision (AP) for every adverb using the sorted classification scores. The average value of all the adverb APs is mAP.

Hit@k: This measures the fraction of test samples that contain at least one of the ground truth labels in the top $k$ predictions. If $r_{a n k}, e$ is the rank of entity $e$ on video $v$ 
(rank 1 gives to the best scoring entity), and $G_{v}$ is the set of ground-truth set for $v$, then the $H i t @ k$ can be written as:

$$
1 /|V| \sum_{v \in V} \vee_{e \in G_{v}} \amalg\left(\operatorname{rank}_{v, e} \leq k\right),
$$

where $\vee$ is logical OR and $\amalg$ is indicator function.

Evaluation We need to define the positive and negative samples first. We treat every person as one sample instead of one video, since there may be more than one player in one video. If the person in the boundingbox is doing the specific action and the action matches the features of one candidate adverb, then, this is a positive sample. If not, then this is a negative sample for that adverb. But what about the condition where the person in the boundingbox is not doing the specific action? Do we just delete it from the sample set or treat it as a negative sample? We treat it as a negative sample. Because models do not have the prior knowledge that whether the person is doing the specific action, we cannot delete it. We realize that there is a possibility that the person in boundingbox who is not doing that specific action is doing another action which is in the action set. we have conducted an experiment and found that the possibility is almost 0 . We randomly choose some boundingboxes belonging to the third group to check whether there is such a risk, and have not found such an example. It is enough to prove that the rate is small enough to ignore.

We use $80 \%$ of the dataset as training set and the remaining as test set. When splitting, we put the videos in the same scene only in one set. Although we deal with the long tail problem, the problem still exists, which is a challenge for HAA recognition approaches.

We use two tasks to evaluate the adverb recognition problem: one is given a video then recognizing the action and adverbs (task 1); the other is given a video and its action categories then recognizing the adverbs (task 2).

Since we have 3 annotators for each case, we use the one which has the closest distance to the predicted value as the ground truth to calculate the evaluation metrics. We use this setting because we believe if a model can recognize HAA like at least one annotator, it is a good model.

\subsection{Attention mechanism}

In this dataset, we treat every person instead of a video as a sample. If we consider human boundingbox regions only, the background context which is useful for HHA recognition may be missed. Therefore, we need to consider the whole image and also tell the model which person in the video to look at. In these experiments, a much more effective attention mechanism is used. We lower the brightness beyond the boundingbox instead of increasing the boundingbox's brightness to reduce the loss of the color saturation in attention area. To avoid fetching in extra edges, we

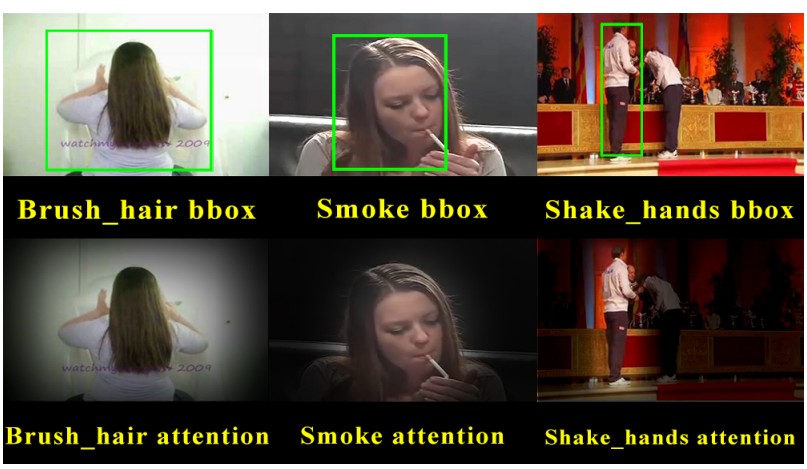

Figure 5. Examples of attention. Top: Row images with boundingboxes. Bottom: Corresponding images after attention process.

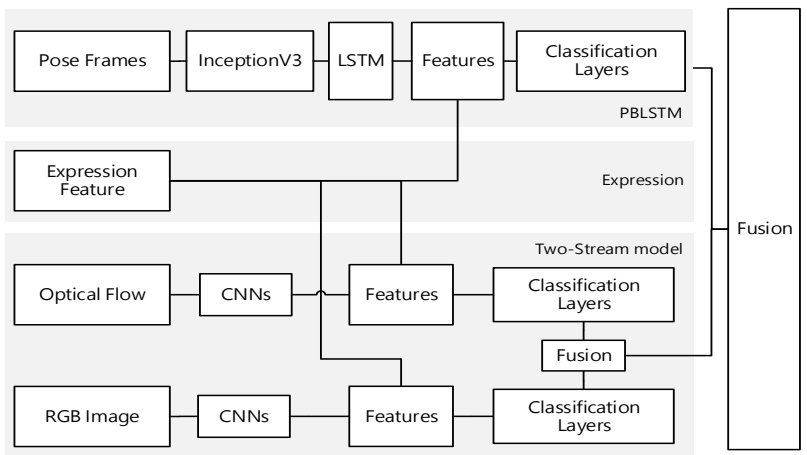

Figure 6. The framework of the Three-Stream Hybrid model. The PBLSTM stream uses pose information; The expression stream uses expression information; And the Two-Stream stream uses optical flow and RGB.

commit smoothing process and some examples are shown in Fig. 5. Smoothing can also deal with the deviation of the MDNet when generating the boundingboxes (like Smoke bbox in Fig. 5). If $\sigma$ is the value to lower the brightness, $B$ is the attention area, $c$ is the center of $B$ and $a$ is the raw value of the point $p$, then the decayed value of $p$ can be written as: $\max (0, a-|p-c| \amalg(p \notin B) \times \sigma)$. In this way, the target person is indicated from the background context.

\subsection{The Three-Stream hybrid model}

We propose a hybrid model using RGB, optical flow, pose and expression information and benchmark it on our ADHA dataset. The framework of the model is shown in Fig. 6.

\subsubsection{Two-Stream Sub-Model}

Two-stream model is really a successful model for action recognition. The two streams are spatial stream and temporal (motion) stream. The former uses frames' RGB information while the latter uses the optical flow information which can show the shifting of every pixel in the video. 


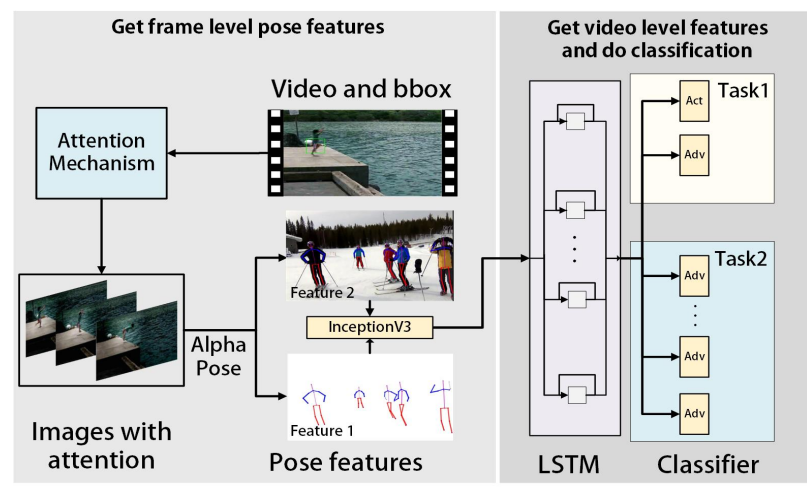

Figure 7. PBLSTM Pipeline. Get the attention pose features first. Then use LSTM (3 layers with 2048 units) to get the fused video level features. For task 1, action and adverb recognition have their own classifiers ("Act" and "Adv" in the figure). For task 2, every action has its own adverb recognition classifiers.

With these two kinds of information, the model can tell what is in the video and how it moves. We refer to [28] to implement the two-stream model. Instead of multi-task learning used in [28] to train the temporal stream, we use cross modality pre-training method proposed in [34] to do weights shape transform so that we can use the ImageNet pre-trained weights in the temporal stream.

We use OpenCV to extract the dense optical flow where the Gunnar Farneback algorithm [9] is used. In this algorithm, the first step is to approximate each neighborhood of both frames by quadratic polynomials, which can be done efficiently using the polynomial expansion transform. Then from observing how an exact polynomial transforms under translation a method to estimate displacement fields from the polynomial expansion coefficients is derived [9]. After getting the optical flow, we sample 10 frames uniformly from the temporal space as the input.

The spatial and motion stream CNNs are pre-trained ResNet101 [12] using ImageNet and fine-tuned on ADHA dataset. Then we put the feature maps provided by these CNNs into different classification layers to do different tasks.

\subsubsection{Pose Based LSTM Sub-Model (PBLSTM)}

LSTM models have achieved great results in action recognition problem. We take an approach similar to [5] to utilize LSTM for adverb recognition. However, unlike that work, we do not use the row video frames as the inputs of the CNN. For adverb recognition, pose is a kind of valuable feature. Although we can gain pose information from the raw frames, they are high dimensional which are not easy to use. So we adopt a state-of-art model [8] to extract pose rending frames and the pure pose frames as the input to reduce the difficulty.
AlphaPose is a system for multi-person 2D pose estimation. The Symmetric STN + SPPE module receives the human bounding boxes obtained by the human detector as input. For each detected bounding box, the corresponding human pose will be predicted by SPPE. And redundent human poses are eliminated by parametric Pose NMS to obtain the final human poses.

We use inceptionV3 [31] pre-trained on ImageNet [3] to extract the feature maps of the poses and we get temporal irrelevant features of each frame. Then put these features into LSTM model to obtain global features and do the recognition. The whole pipeline of the PBLSTM is shown in Fig.7

We set the number of stacked LSTM layers and the number of the hidden units in each layer as the hyperparameters. And the experiments show that 3 layers with 2048 units in them achieve the best performance.

Because in the dataset all the videos are short videos with length around $5 \mathrm{~s}$ ( $3 \mathrm{fps}$ ), we set the maximum number of frames to be 30 which means that the LSTM model is unrolled for 30 iterations. Although a larger unroll number leads to a better performance, it has much lower efficiency.

For task 1, we need to recognize actions and adverbs simultaneously. We set the classification layers into two parts, one for action recognition, the other for adverb recognition. They share the same LSTM layers's weights. For task 2, each action has their own classification layers to do the adverb recognition task and they also share the LSTM layers. The input of the LSTM model is the feature maps of the pose image, and we use two kinds of pose images: one (Feature 1) is pure pose images which only show the human skeletons without background, the other (Feature 2) is the skeletons rendered with the RGB images. There is a problem that whether the RGB information will provide more useful information or more noises for the HAA recognition.

\subsubsection{Using Expression Knowledge}

As discussed earlier, expression recognition has some intersection with adverb recognition. Although they are different problems with different emphasis, we can use the expression as an extra information to improve the performances of the models. We adopt the model [7] which is the winner of EmotiW2016 [4]. It uses a C3D, CNN, RNN hybrid networks.

After getting the expression labels of each video, we use it as another feature and combine them with the CNN features to get the final adverb recognition results. We expect that with the expression information, the classifier will do a better job on adverbs about emotion.

\subsubsection{Fusion the Sub Models}

We will show the result of the sub-models in next part. For each sub model we choose the best settings and add the ex- 


\begin{tabular}{|c|c|c|c|c|c|c|}
\hline \multirow{2}{*}{} & \multicolumn{2}{|c|}{ mAP } & \multicolumn{2}{c|}{ Hit@1 } & \multicolumn{2}{c|}{ Hit@5 } \\
\cline { 2 - 7 } & Act & Adv & Act & Adv & Act & Adv \\
\hline \hline T1-F1 & 6.968 & 6.413 & 36.482 & 38.633 & 66.776 & 73.898 \\
T1-F1-e & 7.732 & $\mathbf{7 . 3 4 7}$ & 40.763 & 43.276 & 70.235 & 74.235 \\
T1-F2 & $\mathbf{7 . 8 8 7}$ & 6.434 & 42.146 & 40.833 & 69.124 & $\mathbf{7 4 . 1 0 0}$ \\
T1-F2-e & 7.886 & 7.153 & $\mathbf{4 5 . 3 1 5}$ & $\mathbf{4 4 . 3 5 6}$ & $\mathbf{7 0 . 4 5 1}$ & 73.892 \\
\hline T2-F1 & - & 6.521 & - & 31.252 & - & 74.903 \\
T2-F1-e & - & 7.362 & - & $\mathbf{3 6 . 3 5 1}$ & - & $\mathbf{7 5 . 3 6 2}$ \\
T2-F2 & - & 7.251 & - & 10.587 & - & 16.560 \\
T2-F2-e & - & $\mathbf{7 . 4 5 9}$ & - & 12.251 & - & 17.358 \\
\hline
\end{tabular}

Table 2. PBLSTM results. "T1-F1" means task 1 with feature 1. “-e" means using expression knowledge. Task 2 doesn't recognize actions so "Act" does not have values in task 2 .

pression features in them. We use average polling method to finally fuse them to get the final results of our hybrid model.

\subsection{Result and Discussion}

First, let's analysis the PBLSTM model's results which are shown in Tab. 2. In the table, "T1-F1" means task 1 using feature 1 mentioned above. And "-e" means using expression knowledge.

We can see that for task 1, using the two kinds of features achieves almost the same results and feature 2 is a little better. While for task 2, feature 1 is much better on Hit@1 and $H i t @ 5$. This reveals that RGB information is useful for action recognition. Just as we discussed above, model can recognize the actions by recognition some specific objects. But when action is confirmed, this RGB information is not so useful for adverb recognition and the high dimension noise will degrades the performance.

When using expression knowledge, $m A P$ values raise about 0.9 point which validates the expectation. And we analysis the results on the 51 adverbs in detail. We find that actually for the adverbs like "heavily" and "slowly" the result is almost the same (for "heavily" $A P$ changes from 5.783 to 5.802 in task 2). While for the adverbs describing the mood and attitude like "happily", the expression really helps (for "happily" $A P$ changes from 6.632 to 7.924 in task 2). So we can tell expression recognition is an important part of adverb recognition.

Comparing task1 and task2, we find that the results for adverb recognition are almost the same, which means the action information is not related to adverb recognition. Therefore, the risk that the model is conducting action recognition while expected to conduct HAA recognition (for example, the model recognizes the adverb "sweetly" due to seeing "kiss") is beingless. This is also the reason why these wonderful models for action recognition do not work well for HAA recognition.

The results of the two-stream model are shown in Tab. 3 "-S" means the spatial stream. "-M" means the motion stream. "-F" is the fusion of the two streams. The analysis discussed above is still suitable for this model.

\begin{tabular}{|c|c|c|c|c|c|c|}
\hline \multirow{2}{*}{} & \multicolumn{2}{|c|}{ mAP } & \multicolumn{2}{c|}{ Hit@ 1 } & \multicolumn{2}{c|}{ Hit@5 } \\
\cline { 2 - 7 } & Act & Adv & Act & Adv & Act & Adv \\
\hline \hline T1-S & 3.806 & 6.246 & 2.140 & 6.140 & 15.400 & 24.850 \\
T1-M & 3.953 & 6.657 & 6.630 & 23.390 & $\mathbf{2 4 . 7 6 0}$ & 53.610 \\
T1-F & 4.126 & 6.792 & 5.870 & 23.190 & 24.120 & $\mathbf{5 5 . 1 4 0}$ \\
T1-F-e & $\mathbf{5 . 6 2 3}$ & $\mathbf{7 . 0 6 4}$ & $\mathbf{7 . 1 6 0}$ & $\mathbf{2 4 . 6 5 0}$ & 24.150 & 54.320 \\
\hline T2-S & - & 6.272 & - & 2.780 & - & 14.960 \\
T2-M & - & 6.251 & - & 4.350 & - & $\mathbf{2 0 . 1 7 0}$ \\
T2-F & - & 6.841 & - & 4.420 & - & 20.140 \\
T2-F-e & - & $\mathbf{7 . 6 2 4}$ & - & $\mathbf{4 . 5 6 0}$ & - & 20.160 \\
\hline
\end{tabular}

Table 3. Two-stream Model results. "-S" means spatial stream. "-M" means motion stream. "-F" means fusion streams. Task 2 doesn't recognize actions so "Act" does not have values in task 2.

\begin{tabular}{|c|c|c|c|c|c|c|}
\hline \multirow{2}{*}{} & \multicolumn{2}{|c|}{ mAP } & \multicolumn{2}{c|}{ Hit@ 1 } & \multicolumn{2}{c|}{ Hit@ 5 } \\
\cline { 2 - 7 } & Act & Adv & Act & Adv & Act & Adv \\
\hline \hline T1-H & 8.103 & 9.235 & 28.135 & 34.292 & 53.329 & 64.325 \\
\hline T2-H & - & 9.738 & - & 27.321 & - & 45.329 \\
\hline
\end{tabular}

Table 4. Hybrid models results. Task 2 doesn't recognize actions so "Act" does not have values in task 2 .

The spatial stream utilizes the RGB information which can tell what is in the video. This information is not so useful for adverb recognition. Even for human, when a person sees an image with a walking human, it is also difficult to tell whether the walking man/woman is free or in a hurry. Compared with spatial stream the motion stream which uses optical flow information shows a much better performance. Obviously, with the speed and the direction of each movement, it is easy for a model to recognize the adverbs.

After fusion the two stream, the performance raises a bit which is not as much as expectation. It is because the spatial stream really achieves a bad performance. In the table, we show the best average fusing result with $20 \%$ weight for spatial stream and $80 \%$ weight for motion stream. We also try the max fusion strategy which is bad, just as we have expected.

Finally the hybrid model results are shown in Tab. 4. The model integrating the information of pose, expression, RGB and optical flow achieve the best performance. But still it is not a satisfactory model and we need further enquiry.

\subsubsection{Influence of Bounding Boxes Correction}

All of the above models use the tracking bounding boxes with human correction which is impossible in real word applications. So we decide to analysis how much this correction will affect the performance.

We choose the best settings of the above models and use the detection and tracking results without human correction to redo the experiments. The result is shown in Tab.5 "-a" means getting the tracking boundingboxes automatically.

We can see that the human correction doesn't affect the results too much. One reason is that the human detection 


\begin{tabular}{|c|c|c|c|c|c|c|}
\hline & \multicolumn{2}{|c|}{ mAP } & \multicolumn{2}{c|}{ Hit@ 1 } & \multicolumn{2}{c|}{ Hit@5 } \\
\cline { 2 - 7 } & Act & Adv & Act & Adv & Act & Adv \\
\hline \hline T1-F-e & 5.623 & 7.064 & 7.160 & 24.650 & 24.150 & 54.320 \\
T1-F-e-a & 5.634 & 6.993 & 7.210 & 24.557 & 24.069 & 54.310 \\
T1-F1-e & 7.732 & 7.347 & 40.763 & 43.276 & 70.235 & 74.235 \\
T1-F1-e-a & 7.729 & 7.336 & 40.49 & 44.282 & 68.124 & 73.125 \\
\hline T2-F-e & - & 7.624 & - & 4.560 & - & 20.160 \\
T2-F-e-a & - & 7.633 & - & 4.558 & - & 20.249 \\
T2-F1-e & - & 7.362 & - & 36.351 & - & 75.362 \\
T2-F1-e-a & - & 7.359 & - & 36.218 & - & 75.349 \\
\hline
\end{tabular}

Table 5. Results with and without human correction. "-a" means the model gets the tracking boundingboxes automatically. Task 2 doesn't recognize actions so "Act" does not have values in task 2.

and tracking algorithms are good enough for the adverb recognition problem. Some tracking-lost problems would not affect the performance. Of course, one must not lose sight of the fact that all mentioned models do not work well.

\section{Conclusions}

We established the first benchmark for recognizing human action adverbs: ADHA. This task is beyond the pattern recognition problems like action recognition. In ADHA, we labeled the actions from a common and describable action set, the adverbs from a semantically complete adverb set, and the human boundingboxes for each person in each video. With ADHA, we benchmarked several outstanding action recognition models. From the result, we can tell that action and adverb recognition have little relativity and using those models achieved unsatisfactory results. Moreover, we propose a hybrid model using RGB, optical flow, pose and expression knowledge and showed that it achieve better results on HAA recognition problem.

\section{References}

[1] S. Abu-El-Haija, N. Kothari, J. Lee, P. Natsev, G. Toderici, B. Varadarajan, and S. Vijayanarasimhan. Youtube-8m: A large-scale video classification benchmark. arXiv preprint, 2016.

[2] M. Davies. The corpus of contemporary American English. BYE, Brigham Young University, 2008.

[3] J. Deng, W. Dong, R. Socher, L.-J. Li, K. Li, and L. FeiFei. Imagenet: A large-scale hierarchical image database. In IEEE Conference on Computer Vision and Pattern Recognition, pages 248-255, 2009.

[4] A. Dhall, R. Goecke, J. Joshi, J. Hoey, and T. Gedeon. Emotiw 2016: Video and group-level emotion recognition challenges. In ACM International Conference on Multimodal Interaction, pages 427-432, 2016.

[5] J. Donahue, L. Anne Hendricks, S. Guadarrama, M. Rohrbach, S. Venugopalan, K. Saenko, and T. Darrell. Long-term recurrent convolutional networks for visual recognition and description. In IEEE conference on computer vision and pattern recognition, pages 2625-2634, 2015.
[6] E. Douglas-Cowie, R. Cowie, I. Sneddon, C. Cox, O. Lowry, M. Mcrorie, J.-C. Martin, L. Devillers, S. Abrilian, A. Batliner, et al. The humaine database: addressing the collection and annotation of naturalistic and induced emotional data. Affective computing and intelligent interaction, pages 488500, 2007.

[7] Y. Fan, X. Lu, D. Li, and Y. Liu. Video-based emotion recognition using cnn-rnn and c3d hybrid networks. In ACM International Conference on Multimodal Interaction, pages 445450, 2016.

[8] H.-S. Fang, S. Xie, Y.-W. Tai, and C. Lu. RMPE: Regional multi-person pose estimation. In ICCV, 2017.

[9] G. Farnebäck. Two-frame motion estimation based on polynomial expansion. Image analysis, pages 363-370, 2003.

[10] R. Girshick. Fast r-cnn. In IEEE international conference on computer vision, pages 1440-1448, 2015.

[11] S. Guadarrama, N. Krishnamoorthy, G. Malkarnenkar, S. Venugopalan, R. Mooney, T. Darrell, and K. Saenko. Youtube2text: Recognizing and describing arbitrary activities using semantic hierarchies and zero-shot recognition. In IEEE international conference on computer vision, pages 2712-2719, 2013.

[12] K. He, X. Zhang, S. Ren, and J. Sun. Deep residual learning for image recognition. In Proceedings of the IEEE conference on computer vision and pattern recognition, pages 770-778, 2016.

[13] S. Ji, W. Xu, M. Yang, and K. Yu. 3d convolutional neural networks for human action recognition. IEEE transactions on pattern analysis and machine intelligence, 35(1):221231, 2013.

[14] A. Karpathy, G. Toderici, S. Shetty, T. Leung, R. Sukthankar, and L. Fei-Fei. Large-scale video classification with convolutional neural networks. In IEEE conference on Computer Vision and Pattern Recognition, pages 1725-1732, 2014.

[15] R. Krishna, K. Hata, F. Ren, L. Fei-Fei, and J. Carlos Niebles. Dense-captioning events in videos. In The IEEE International Conference on Computer Vision (ICCV), Oct 2017.

[16] M. Kristan, J. Matas, A. Leonardis, M. Felsberg, L. Cehovin, G. Fernandez, T. Vojir, G. Hager, G. Nebehay, and R. Pflugfelder. The visual object tracking vot2015 challenge results. In The IEEE International Conference on Computer Vision (ICCV) Workshops, December 2015.

[17] H. Kuehne, H. Jhuang, E. Garrote, T. Poggio, and T. Serre. Hmdb: a large video database for human motion recognition. In IEEE International Conference on Computer Vision (ICCV), pages 2556-2563, 2011.

[18] I. Laptev. On space-time interest points. International journal of computer vision, 64(2-3):107-123, 2005.

[19] I. Laptev, M. Marszalek, C. Schmid, and B. Rozenfeld. Learning realistic human actions from movies. In IEEE Conference on Computer Vision and Pattern Recognition $(C V P R)$, pages 1-8, 2008.

[20] S. Maji, L. Bourdev, and J. Malik. Action recognition from a distributed representation of pose and appearance. In IEEE Conference on Computer Vision and Pattern Recognition (CVPR), pages 3177-3184, 2011. 
[21] H. Nam and B. Han. Learning multi-domain convolutional neural networks for visual tracking. CoRR, abs/1510.07945, 2015.

[22] A. Newell, K. Yang, and J. Deng. Stacked hourglass networks for human pose estimation. In European Conference on Computer Vision, pages 483-499, 2016.

[23] H.-W. Ng, V. D. Nguyen, V. Vonikakis, and S. Winkler. Deep learning for emotion recognition on small datasets using transfer learning. In ACM on international conference on multimodal interaction, pages 443-449, 2015.

[24] J.-Y. Pan, H.-J. Yang, C. Faloutsos, and P. Duygulu. Gcap: Graph-based automatic image captioning. In Computer Vision and Pattern Recognition Workshop, 2004. CVPRW'04. Conference on, pages 146-146, 2004.

[25] A. Petrosino and K. Gold. Toward fast mapping for robot adjective learning. In 2010 AAAI Fall Symposium Series, 2010.

[26] J. Redmon and A. Farhadi. Yolo9000: better, faster, stronger. arXiv preprint, 2016.

[27] S. Ren, K. He, R. Girshick, and J. Sun. Faster r-cnn: Towards real-time object detection with region proposal networks. In Advances in neural information processing systems, pages 91-99, 2015.

[28] K. Simonyan and A. Zisserman. Two-stream convolutional networks for action recognition in videos. In Advances in neural information processing systems, pages 568-576, 2014.

[29] K. Soomro, A. R. Zamir, and M. Shah. Ucf101: A dataset of 101 human actions classes from videos in the wild. arXiv preprint, 2012.

[30] N. Srivastava, E. Mansimov, and R. Salakhudinov. Unsupervised learning of video representations using lstms. In International Conference on Machine Learning, pages 843-852, 2015.

[31] C. Szegedy, V. Vanhoucke, S. Ioffe, J. Shlens, and Z. Wojna. Rethinking the inception architecture for computer vision. In IEEE Conference on Computer Vision and Pattern Recognition, pages 2818-2826, 2016.

[32] M. Valstar and M. Pantic. Induced disgust, happiness and surprise: an addition to the mmi facial expression database. In Workshop on EMOTION (satellite of LREC): Corpora for Research on Emotion and Affect, page 65, 2010.

[33] H. Wang, A. Kläser, C. Schmid, and C.-L. Liu. Action recognition by dense trajectories. In IEEE Conference on Computer Vision and Pattern Recognition (CVPR), pages 31693176, 2011.

[34] L. Wang, Y. Xiong, Z. Wang, Y. Qiao, D. Lin, X. Tang, and L. Van Gool. Temporal segment networks: Towards good practices for deep action recognition. In European Conference on Computer Vision, pages 20-36, 2016.

[35] L. White. Adverb placement in second language acquisition: Some effects of positive and negative evidence in the classroom. Interlanguage studies bulletin (Utrecht), 7(2):133$161,1991$.

[36] Z. Wu, X. Wang, Y.-G. Jiang, H. Ye, and X. Xue. Modeling spatial-temporal clues in a hybrid deep learning framework for video classification. In ACM international conference on Multimedia, pages 461-470, 2015.
[37] J. Xu, T. Mei, T. Yao, and Y. Rui. Msr-vtt: A large video description dataset for bridging video and language. In IEEE Conference on Computer Vision and Pattern Recognition, pages 5288-5296, 2016.

[38] K. Xu, J. Ba, R. Kiros, K. Cho, A. Courville, R. Salakhudinov, R. Zemel, and Y. Bengio. Show, attend and tell: Neural image caption generation with visual attention. In International Conference on Machine Learning, pages 2048-2057, 2015.

[39] J. Yue-Hei Ng, M. Hausknecht, S. Vijayanarasimhan, O. Vinyals, R. Monga, and G. Toderici. Beyond short snippets: Deep networks for video classification. In IEEE conference on computer vision and pattern recognition, pages 4694-4702, 2015. 